\title{
Application of digital technologies to leprosy programmes
}

\author{
Geoff Warne ${ }^{\mathrm{a}} \&$ Monty Mukhier ${ }^{\mathrm{a}}$ \\ ${ }^{a}$ International Federation of Anti-Leprosy Associations (ILEP), Route du \\ Nant-d'avril 150, 1217 Meyrin, Switzerland
}

Submitted and accepted 7 May 2021

\begin{abstract}
Summary Rapid advances in digital technologies have major potential impacts on the way leprosy programmes are undertaken. This report describes the way ILEP Member associations are deploying innovative technologies in mapping, diagnostics, health information systems and patient management, and notes the need to continue to stay abreast of new technology thinking to improve programmes for the ultimate benefit of people affected by leprosy.
\end{abstract}

Keywords: Knowledge, direct and indirect health information technology

\section{Introduction}

The International Federation of Anti-Leprosy Associations (ILEP) holds periodic conferences to enable Member associations to work collaboratively on matters of mutual interest and concern. The September 2020 conference was in two halves. The topic for the first half conference, which is the subject of this report, was the opportunities for use of new digital technologies to improve leprosy services. The second half, which will be reported separately, tackled the challenges arising from the loss of leprosy expertise worldwide. 165 people participated in this virtual conference, more than 90 of whom were from leprosy-endemic countries.

\section{Innovative technologies}

The aims of the conference were to learn from one another's experiences in using digital applications, compare the benefits of different approaches and apps, and gain awareness of potential new digital applications that are currently in development. Digital approaches were clustered into four categories: mapping, diagnostic tools, health information systems and patient management. Each session consisted of a thematic introduction, a set of presentations, and a plenary discussion. An index of presenters, with contact details, is in Table 1. Recordings of all presentations can be viewed using the links in Table 2 .

Correspondence to: Geoff Warne, ILEP (e-mail: gwarne@ilepfederation.org) 
Table 1. Presenters and contact details

\begin{tabular}{lll}
\hline Presentation & Presenter & Contact \\
\hline Mapping overview & Paul Saunderson & psaunderson@ leprosy.org \\
Mapping (i) & Leslie Zolman & lzolman@leprosy.org \\
Mapping (ii) & Anneke Taal & a.taal@nlrinternational.org \\
Mapping (iii) & Shyamala Anand & shyamala@leprosy.org \\
& Suresh Munnuswami & s.munuswamy@iiphh.org \\
Mapping (iv) & Ashish Wagh & Ashish.wagh@ damienfoundation.in \\
Mapping (v) & Stéphanie Ramboarina & sramboa@ gmail.com \\
Diagnostic tools overview & Sundeep Chaitanya & schaitanya@leprosy.org \\
Diagnostic tools (i) & Lucy Setian & lucy.setian@ @ovartis.com \\
Diagnostic tools (ii) & Madhusmita Das & deenaanddavid@gmail.com \\
Diagnostic tools (iii) & Estelle Marion & estelle.marion@inserm.fr \\
Diagnostic tools (iv) & Liesbeth Mieras & L.Mieras@nlrinternational.org \\
H.I.S. overview & Sunil Anand & sanand@leprosy.org \\
H.I.S. (i) & John Kurian George & john@fairmedindia.in \\
H.I.S. (ii) & Pemmaraju Rao & pemmarajuv@who.int \\
H.I.S. (iii) & Duane Hinders & D.Hinders@ @lrinternational.org \\
H.I.S. (iv) & Joydeepa Darlong & joydeepa.darlong@leprosymission.in \\
& Gajananda Bhandari & gajanandab@tlmnepal.org \\
H.I.S. (v) & Jose Manikkathan & jose@aifoindia.org \\
Patient management overview & Charles Mackenzie & cmackenzie@taskforce.org \\
Patient management (i) & Satish Paul & sathishpaul77@gmail.com \\
Patient management (ii) & Nimer Ortuno Gutierrez & Nimer.OrtunoGutierrez@damiaanactie.be \\
Patient management (iii) & Satish Paul & sathishpaul77@ gmail.com \\
Patient management (iv) & Léa Guignard & 1.guignard@enablement.nl \\
Patient management (v) & John Kurian George & john@fairmedindia.in \\
\hline
\end{tabular}

Table 2. Recordings of presentations

\begin{tabular}{ll}
\hline Mapping & Mapping presentations on ILEP Dropbox \\
Diagnostic tools & Diagnostic Tools presentations on ILEP Dropbox \\
Health information systems & Health Information Systems presentations on ILEP Dropbox \\
Patient management & Patient Management presentations on ILEP Dropbox \\
\hline
\end{tabular}

\section{MAPPING}

The session was introduced by Dr Paul Saunderson with an overview of the development of mapping technologies. The presentations were on:

(i) The use of mapping technologies in leprosy and other NTDs, supporting governments to use better data for planning and programme management (AIM Initiative).

(ii) The set of mapping and cluster identification technologies used in the PEP++ leprosy project to target blanket preventive chemotherapy campaigns (NLR).

(iii) Ecosystem of AI-enabled technologies for comprehensive, economical at-home services for long-term management of leprosy, LF and WASH (American Leprosy Missions).

(iv) Use of the mobile app Open Data Kit to collect GIS data on index cases and contacts, enabling data analysis and clear visual representation (Damien Foundation Belgium).

(v) Recording and graphical display of personal, diagnostic and GPS data for new leprosy cases in the PEOPLE active case-finding project (Fondation Raoul Follereau). 
DIAGNOSTIC TOOLS

The session started with a description by Dr Sundeep Chaitanya of work in progress on a target product profile for a leprosy diagnostic test. The presentations were on:

(i) The AI4Leprosy project which aims to develop an easy-to-use AI powered solution to accelerate diagnosis of leprosy with skin images (Novartis).

(ii) Use of the Biomeme platform towards a field-friendly PCR-based early diagnostic tool to detect subclinical infection among household contacts and the general population (Karigiri).

(iii) BU-LABNET, a 10-country laboratory network providing quality assessment and supporting standardised procedures for PCR-based diagnosis of Buruli ulcer (Fondation Raoul Follereau).

(iv) Overview and development plans for the SkinApp, which supports health workers to learn about, diagnose and manage skin diseases including skin-related NTDs (NLR).

\section{HEALTH INFORMATION SYSTEMS}

The session was introduced with an overview by Dr Sunil Anand. The presentations were on:

(i) A web-based hospital information system that captures and enables analysis of information about the medical services provided to patients at tertiary hospitals in India (FAIRMED).

(ii) Use of DHIS2 to capture aggregate leprosy data (at national and sub-national levels) and individual case-based information (WHO Global Leprosy Programme).

(iii) Selection process and assessment of the strengths and weaknesses of the electronic data capture system REDCap (NLR).

(iv) Central hospital information system to improve intra-disciplinary collaboration between departments in leprosy hospitals and enable better reporting (The Leprosy Mission).

(v) Use of cloud-based Goonjan management information system to record patient data, training and campaign data to enable digitised work planning and reporting (AIFO).

\section{PATIENT MANAGEMENT}

The session started with a wide overview by Dr Charles Mackenzie on the impact of newera digital developments on the entire range of aspects of patient management, including the potential of AI and Big Data for better assessment and decision-making. The presentations were on:

(i) A study using Bluetooth technology in a sensory feedback system to predict and prevent peak plantar pressures on anaesthetic feet (Karigiri).

(ii) Use by patients of GPS-tracker devices to trace mobility, assess health-seeking behaviour and support rationalised contact tracing (Damien Foundation Belgium).

(iii) Supply to patients of mobile handsets enabling personalised two-way flow of information between patients and the hospital (Karigiri).

(iv) Use and development of RehApp, a digital tool for field workers that work with persons with disabilities (Enablement/The Leprosy Mission).

(v) Tablet-based tool that captures information, including images, about health and disability services provided to people in the field, enabling prompt follow up and referral (FAIRMED). 


\section{Discussion and next steps}

This conference followed a brief 'taster' session in 2018, and the technology advances over the intervening two years were very striking. Demonstrating a new technology, and fielding questions about it, by Zoom was challenging compared with the immediacy of a face to face conference, though some presenters managed a 'show and tell' session very effectively. On the other hand, holding the conference virtually enabled almost three times more attendees that would otherwise have been able to participate.

Of the 44 attendees that completed a post-conference survey, $89 \%$ said that they intended to use one or more of the technologies profiled to improve their services, $75 \%$ intended to follow up with the presenters to get further information, and $82 \%$ were interested to listen to the recordings of the sessions they had not been able to attend.

It was particularly valuable to combine these informative sessions about how current technologies are being used, with sessions that looked outward at digital developments in the wider world, such as AI, voice recognition, machine learning and virtual agents, that may impact leprosy services in the years ahead. ILEP will consider hosting further conferences on new digital technologies at regular intervals, to enable Member associations and other stakeholders to stay abreast of new thinking and to continue to learn from one another's experiences of putting technologies into action for the ultimate benefit of people affected by leprosy.

\section{Acknowledgements}

The authors wish to thank all the presenters and the session coordinators. Sincere appreciation to Sunil Anand, Sundeep Chaitanya, Jannine Ebenso and Paul Watson for their support and advice as members of the conference planning team. 